\title{
Accuracy of detecting enlargement of aneurysms using different MRI modalities and measurement protocols
}

\author{
Daichi Nakagawa, MD, PhD,,2 Yasunori Nagahama, MD,' Bruno A. Policeni, MD, ${ }^{3}$ \\ Madhavan L. Raghavan, PhD, ${ }^{2}$ Seth I. Dillard, PhD, ${ }^{2}$ Anna L. Schumacher, PhD, ${ }^{2}$ \\ Srivats Sarathy, MS, ${ }^{2}$ Brian J. Dlouhy, MD, ${ }^{1}$ Saul Wilson, MD, ${ }^{1}$ Lauren Allan, DO, ${ }^{4}$ \\ Henry H. Woo, MD, ${ }^{5}$ John Huston III, MD, ${ }^{6}$ Harry J. Cloft, MD, PhD, ${ }^{6}$ Max Wintermark, MD, ${ }^{7}$ \\ James C. Torner, PhD, ${ }^{8}$ Robert D. Brown Jr., MD, MPH, ${ }^{9}$ and David M. Hasan, MD ${ }^{1}$
}

Departments of ${ }^{1}$ Neurosurgery, ${ }^{3}$ Radiology, and ${ }^{8}$ Epidemiology, University of lowa Hospitals and Clinics, lowa City; ${ }^{2}$ Department of Biomedical Engineering, University of lowa, Seamans Center for the Engineering Arts and Sciences, lowa City; ${ }^{4}$ Department of General Surgery, Mercy Medical Center, Des Moines, lowa; ${ }^{5}$ Department of Neurosurgery, Stony Brook University, Stony Brook, New York; Departments of ${ }^{6}$ Radiology and ${ }^{9}$ Neurology, Mayo Clinic, Rochester, Minnesota; and ${ }^{7}$ Department of Radiology, Stanford University Medical School, Palo Alto, California

OBJECTIVE Aneurysm growth is considered predictive of future rupture of intracranial aneurysms. However, how accurately neuroradiologists can reliably detect incremental aneurysm growth using clinical MRI is still unknown. The purpose of this study was to assess the agreement rate of detecting aneurysm enlargement employing generally used MRI modalities.

METHODS Three silicone flow phantom models, each with 8 aneurysms of various sizes at different sites, were used in this study. The aneurysm models were identical except for an incremental increase in the sizes of the 8 aneurysms, which ranged from $0.4 \mathrm{~mm}$ to $2 \mathrm{~mm}$. The phantoms were imaged on 1.5-T and 3-T MRI units with both time-of-flight (TOF) and contrast-enhanced MR angiography. Three independent expert neuroradiologists measured the aneurysms in a blinded manner using different measurement approaches. The individual and agreement detection rates of aneurysm enlargement among the 3 experts were calculated.

RESULTS The mean detection rate of any increase in any aneurysmal dimension was $95.7 \%$. The detection rates of the 3 observers (observers $A, B$, and C) were $98.0 \%, 96.6 \%$, and $92.7 \%$, respectively $(p=0.22)$. The detection rates of each MRI modality were $91.3 \%$ using $1.5-\mathrm{T}$ TOF, $97.2 \%$ using $1.5-\mathrm{T}$ with $\mathrm{Gd}, 95.8 \%$ using $3.0-\mathrm{T}$ TOF, and $97.2 \%$ using 3.0-T with $\mathrm{Gd}(p=0.31)$. On the other hand, the mean detection rate for aneurysm enlargement was $54.8 \%$. Specifically, the detection rates of observers $A, B$, and $C$ were $49.0 \%, 46.1 \%$, and $66.7 \%$, respectively $(p=0.009)$. As the incremental enlargement value increased, the detection rate for aneurysm enlargement increased. The use of 1.5-T Gd improved the detection rate for small incremental enlargement (e.g., $0.4-1 \mathrm{~mm})$ of the aneurysm $(p=0.04)$. The location of the aneurysm also affected the detection rate for aneurysm enlargement $(p<0.0001)$.

CONCLUSIONS The detection rate and interobserver agreement were very high for aneurysm enlargement of 0.4-2 $\mathrm{mm}$. The detection rate for at least 1 increase in any aneurysm dimension did not depend on the choice of MRI modality or measurement protocol. Use of Gd improved the accuracy of measurement. Aneurysm location may influence the accuracy of detecting enlargement.

https://thejns.org/doi/abs/10.3171/2017.9.JNS171811

KEY WORDS aneurysm growth; detection; magnetic resonance angiography; magnetic resonance imaging; aneurysm size; phantom model; vascular disorders

ABBREVIATIONS BA = basilar artery; ICA = internal carotid artery; MCA = middle cerebral artery; MRA = MR angiography; TOF = time of flight.

SUBMITTED July 24, 2017. ACCEPTED September 11, 2017.

INCLUDE WHEN CITING Published online March 9, 2018; DOI: 10.3171/2017.9.JNS171811. 
$\mathrm{S}$ EVERAL risk factors that predict increased risk of aneurysmal subarachnoid hemorrhage have been reported. $3,6,8,9,11,14-16,18,22,25$ Among these factors, aneurysm growth is considered one of the most reliable predictors of increased risk of aneurysmal subarachnoid hemorrhage. . $^{3,8,11,14,22}$ Thus, it is crucial for clinicians to reliably measure intracranial aneurysms and accurately detect their growth. $2,8,13,18$

Imaging intracranial aneurysms can be done using several imaging modalities that are commercially available, such as CT angiography, ${ }^{2,13,23,24}$ MR angiography (MRA),, 2, , ${ }^{8,24}$ digital subtraction angiography, ${ }^{10,12,26}$ and 3D rotational angiography. ${ }^{1,4,17,20,21}$ It is well known that 3D rotational angiography provides a more accurate depiction of the anatomical details of the aneurysm dome and neck. However, it is invasive and may require more contrast agent.

On the other hand, MRA is a minimally invasive imaging modality that is crucial for imaging intracranial aneurysms in clinical practice. , $, 7,24^{\text {Additionally, how }}$ accurately neuroradiologists can reliably detect small incremental growth of aneurysms using commercially available MRI modalities is still unknown.

The purpose of this study was to evaluate the accuracy of detecting aneurysm growth using 1.5-T and 3.0-T with both time-of-flight (TOF) and contrast-enhanced MRA. Aneurysm flow phantom models were specifically used to accurately enlarge the sizes of aneurysms in small increments.

\section{Methods}

\section{Aneurysm Phantoms and Flow Simulator}

Three silicone flow phantom models of the complete circle of Willis with 8 aneurysms of various sizes in different locations were created using a 3D printer (Vascular Simulations LCC). Marginal errors in aneurysm size were within $\pm 0.2 \mathrm{~mm}$. The baseline aneurysms were replicas of true human aneurysms that were recreated with 3D rotational angiography. Each phantom model had 4 inlets (2 internal carotid arteries [ICAs] and 2 vertebral arteries) and 3 outlets ( 2 anterior circulations from both the left and right side and 1 posterior circulation from both the left and right posterior cerebral arteries). Each phantom model contained an aneurysm at each of the following 8 locations: anterior communicating artery, right middle ce-

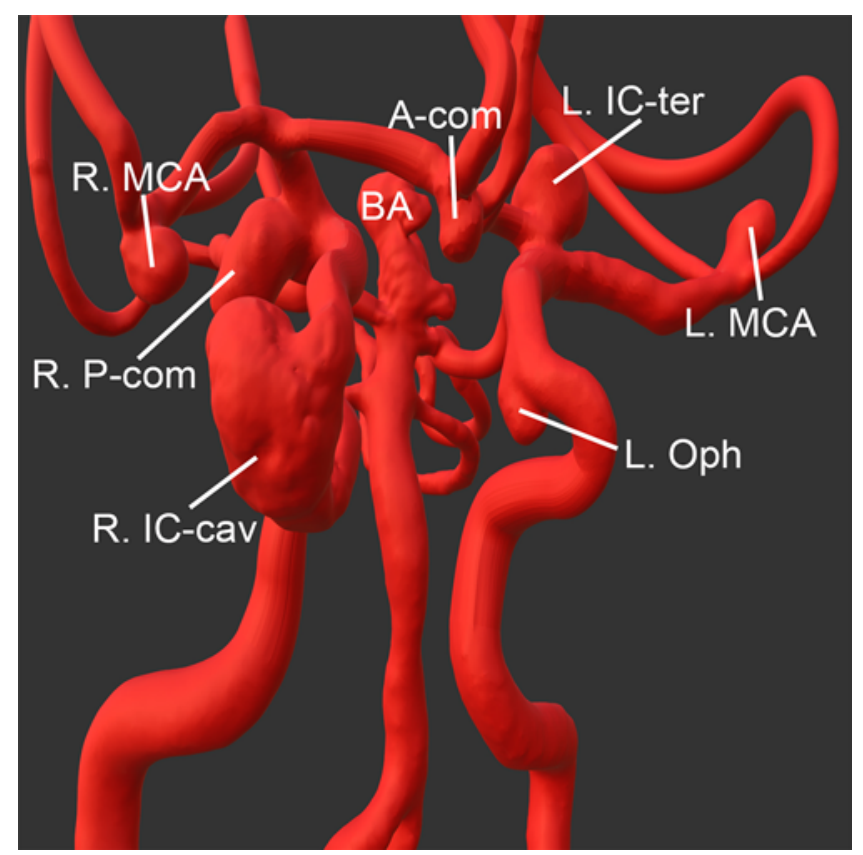

FIG. 1. Silicone flow phantom with 8 aneurysms of various sizes. A-com = anterior communicating artery; IC-cav = cavernous segment of the ICA; IC-ter = terminus segment of the ICA; L. = left; Oph = ophthalmic artery; $\mathrm{P}$-com = posterior communicating artery; $\mathrm{R} .=$ right. Figure is available in color online only.

rebral artery (MCA), left MCA, left ICA-ophthalmic artery, right posterior communicating artery, left terminal of the ICA, basilar artery (BA), and the cavernous portion of the right ICA (Fig. 1). From phantom 1, the 8 aneurysms were enlarged between 0.4 and $1.0 \mathrm{~mm}$ to create phantom 2. Likewise, from phantom 2 , the aneurysms were again enlarged between 0.4 and $1.0 \mathrm{~mm}$ to create phantom 3 . Details of the baseline aneurysm sizes (phantom 1), locations, directions of growth, incremental growths, and subsequent aneurysm sizes after growth (phantoms 2 and 3) are described in Table 1. All phantoms were emerged in gel to minimize susceptibility artifacts from the air interfaces. To simulate blood flow through the phantom model, a flow loop was set up. In the flow loop, the inlet and outlet tank was filled with $1 \mathrm{~L}$ of water without contrast for the TOF MRA sequences and with Gd contrast agent for

TABLE 1. Real aneurysm height and width in the 3 phantoms

\begin{tabular}{|c|c|c|c|c|c|c|c|}
\hline \multirow[b]{2}{*}{ Location } & \multicolumn{2}{|c|}{ Phantom 1} & \multicolumn{2}{|c|}{ Phantom 2} & \multicolumn{2}{|c|}{ Phantom 3} & \multirow[b]{2}{*}{ Enlarged } \\
\hline & Width & Height & Width & Height & Width & Height & \\
\hline Anterior communicating artery & 4.07 & 3.04 & 4.07 & 3.44 & 4.07 & 3.84 & Height by 0.4 \\
\hline Rt MCA & 3.72 & 4.10 & 3.72 & 4.50 & 3.72 & 4.90 & Height by 0.4 \\
\hline Lt MCA & 4.83 & 5.60 & 5.33 & 5.60 & 5.83 & 5.60 & Width by 0.5 \\
\hline Lt ICA-ophthalmic artery & 6.00 & 3.56 & 6.60 & 3.56 & 7.20 & 3.56 & Width by 0.6 \\
\hline Rt posterior communicating artery & 6.80 & 8.90 & 7.50 & 9.60 & 8.20 & 10.3 & Width \& height by 0.7 \\
\hline Terminus of the It ICA & 7.90 & 7.21 & 8.70 & 8.01 & 9.60 & 8.81 & Width \& height by 0.8 \\
\hline $\mathrm{BA}$ & 8.76 & 10.78 & 9.66 & 11.68 & 10.56 & 12.58 & Width \& height by 0.9 \\
\hline Cavernous portion of the rt ICA & 9.83 & 15.34 & 10.83 & 16.34 & 11.83 & 17.34 & Width \& height by 1.0 \\
\hline
\end{tabular}

All values are shown in millimeters. 
TABLE 2. MRI protocols

\begin{tabular}{lcccc}
\hline \multicolumn{1}{c}{ Variable } & 1.5-T TOF & 1.5 -T Gd & 3.0 -T TOF & $3.0-$ T Gd \\
\hline Matrix & $256 \times 256$ & $320 \times 256$ & $320 \times 320$ & $384 \times 384$ \\
\hline Slice thickness, $\mathrm{mm}$ & 0.7 & 0.7 & 0.5 & 0.8 \\
\hline Spatial resolution, $\mathrm{mm}$ & $0.7 \times 0.7 \times 0.7$ & $0.63 \times 0.78 \times 0.7$ & $0.69 \times 0.69 \times 0.5$ & $0.58 \times 0.58 \times 0.8$ \\
\hline TR, msec & 27 & 3.23 & 21 & 3.32 \\
\hline TE, msec & 7.15 & 1.21 & 3.43 & 1.21 \\
\hline
\end{tabular}

the contrast-enhanced MRA sequences. Water with and without Gd was pumped from the tank to the 4 inlets of the phantom using a peristatic pump to maintain an approximate continuous steady flow of $750 \mathrm{ml} / \mathrm{min}$ at the inlet. Before the experiment, each phantom was visually inspected to make sure it did not demonstrate any abnormal behaviors, including any deformations, cracks, or size changes.

\section{Imaging Modalities}

Images were obtained on each phantom model using 1.5-T (Magentron Avanto, Siemens) and 3.0-T (Signa MR750, GE) units at the University of Iowa Hospitals and Clinics and employing standard clinical scanning protocols. All images were translated to maximum intensity projection. The window level and window width of the maximum intensity projection were determined at the discretion of the radiology personnel. Details of the MRI protocols are described in Table 2.

\section{Evaluation of Aneurysm Size}

Three independent and expert neuroradiologists (J.H., H.J.C., and M.W.) measured the sizes of these aneurysms. The observers were not allowed to look at the images of the 3 phantoms side-by-side. To evaluate aneurysm size, 2 neuroradiologists (observers A and B) used the International Study of Unruptured Intracranial Aneurysms measurement protocol, ${ }^{5}$ which first determines and measures the maximum internal dimension of the aneurysm. Then, orthogonal to this maximum measurement, a maximum transverse diameter was measured. Finally, the remaining ordinal direction was measured. Observer $\mathrm{C}$ measured the height and width of the aneurysms. The maximum height was defined as the maximum length of the aneurysm perpendicular to the aneurysm neck plane. Likewise, maximum width was defined as the maximum length of the aneurysm in any direction that was not the maximal height. This difference in the aneurysm measurement technique allowed us to determine whether aneurysm enlargement was more accurately detected by either approach.

\section{Detection of Aneurysm Enlargement}

Detection of an enlarged aneurysm dimension was defined as an increase of $\geq 0.1 \mathrm{~mm}$ in the measurement of that dimension. Likewise, detection of a decreased aneurysm dimension was defined as a decrease of $\geq 0.1 \mathrm{~mm}$ in the measurement of that dimension. Based on that, aneurysms that were read as having enlargement in at least 1 dimension and no change in the other dimensions were considered enlarged aneurysms. Aneurysms that were determined by the observers to have enlargement in at least 1 dimension and at least 1 decrease in the other dimensions were considered pseudo-enlarged aneurysms. Aneurysms that were read as having a decrease in at least 1 dimension and no change in the other dimensions were considered shrunken aneurysms. Stable aneurysms were those that had no changes noted by the observers in any aneurysm dimension. The summary of the classification system for aneurysm changes is described in Fig. 2.

\section{Statistical Analysis}

The statistical analysis and graphic display of data were performed using GraphPad software (version 7.00 for Windows). To compare percentages among observers, the Fisher exact or chi-square test with the paired-comparison test was conducted. In all analyses, $\mathrm{p}<0.05$ was considered to indicate a significant difference.

\section{Results}

There were 768 measurements in total to be determined. However, due to imaging quality, 1 reader (observer A) found it difficult to determine the dimensions needed to record the aneurysm size; thus, only 752 measurements were obtained. Observer A deemed the imaging quality of the aneurysms in question to be suboptimal.

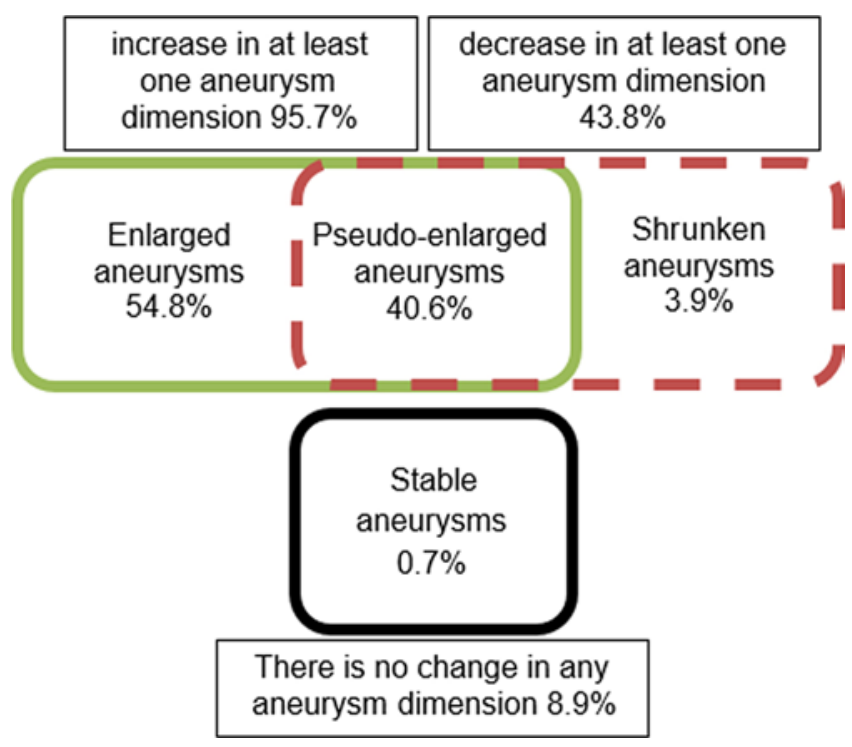

FIG. 2. Summary of the classification system for aneurysm change and the results of this study. Figure is available in color online only. 


\section{Interobserver Variability}

Detection Rate of Increased Size in at Least 1 Aneurysm Dimension

The mean detection rate of increased size in at least 1 aneurysm dimension for all MRI modalities was $95.7 \%$ (Fig. 2). The detection rates of observers A, B, and $\mathrm{C}$ were $98.0 \%, 96.6 \%$, and $92.7 \%$, respectively ( $\mathrm{p}=0.22$; Table 3 ). The detection rates of increased size in at least 1 aneurysm dimension for each MRI modality were $91.3 \%$ for $1.5-\mathrm{T}$ TOF, $97.2 \%$ for $1.5-\mathrm{T}$ Gd-enhanced, $95.8 \%$ for $3.0-\mathrm{T}$ TOF, and $97.2 \%$ for 3.0-T Gd-enhanced images. Even though there was an increase in the rate of detection of aneurysm enlargement with the use of 3.0-T Gd when compared with 1.5-T TOF, this difference was not significant $(\mathrm{p}=$ 0.31 ) between these MRI modalities after analysis according to the Fisher exact test.

\section{Detection Rate of Aneurysm Enlargement}

Because we defined aneurysm enlargement as an increase in at least 1 of any of the dimensions and no decrease in any of the other dimensions, the mean detection rate of aneurysm enlargement for the 3 observers was $54.8 \%$ (Fig. 2). Specifically, the detection rates for observers A, B, and C were $49.0 \%, 46.1 \%$, and $66.7 \%$, respectively $(\mathrm{p}=0.009)$, meaning there were significant differences in interobserver agreement for aneurysm enlargement between observer A versus $C(p=0.02)$ and observer B versus $C(p=0.005)$ according to the paired-comparison test. When considering the 4 different MRI modalities (1.5-T TOF, 1.5-T Gd, 3.0-T TOF, and 3.0-T Gd), there was no significant difference between interobserver agreement in aneurysm enlargement (Table 4).

\section{Detection Rate of Any Decrease in Any Aneurysm Dimension}

The detection rate of any decrease in any aneurysm dimension for all MRI modalities was $43.8 \%$ (Fig. 2). Specifically, the detection rates of observers $\mathrm{A}, \mathrm{B}$, and $\mathrm{C}$ were $50.0 \%, 51.7 \%$, and $30.2 \%(\mathrm{p}=0.004)$, respectively, meaning there were significant differences in interobserver agreement between observer $A$ versus $C(p=0.008)$ and observer $B$ versus $C(p=0.005)$ according to the pairedcomparison test. When considering the 4 different MRI modalities, there was no significant difference in interobserver agreement $(\mathrm{p}=0.07$ for $1.5-\mathrm{T}$ TOF, $\mathrm{p}=0.08$ for 1.5-T Gd, $\mathrm{p}=0.70$ for 3.0-T TOF, and $\mathrm{p}=0.13$ for 3.0-T $\mathrm{Gd})$. The detection rates for any decrease in any aneurysm

TABLE 3. Results of the Fisher exact test with the pairedcomparison test for interobserver variability in the detection rates of increased size in at least 1 aneurysm dimension

\begin{tabular}{lcccc}
\hline & \multicolumn{3}{c}{ Detection Rate } & \\
\cline { 2 - 4 } Modality & Observer A & Observer B & Observer C & p Value \\
\hline Total & 98.0 & 96.6 & 92.7 & 0.22 \\
\hline 1.5-T TOF & 100 & 75.0 & 83 & 0.13 \\
\hline 1.5-T Gd & 95.8 & 100 & 100 & $>0.99$ \\
\hline 3.0-T TOF & 95.8 & 100 & 95.8 & $>0.99$ \\
\hline 3.0-T Gd & 100 & 100 & 91.7 & 0.32 \\
\hline
\end{tabular}

All values are shown as percentages unless indicated otherwise. dimension for each MRI modality were $50.7 \%$ for $1.5-\mathrm{T}$ TOF, $38.6 \%$ for $1.5-\mathrm{T} \mathrm{Gd}, 48.6 \%$ for $3.0-\mathrm{T} \mathrm{TOF}$, and $37.5 \%$ for $3.0-\mathrm{T} \mathrm{Gd}(\mathrm{p}=0.27)$.

\section{Detection Rate of Aneurysm Shrinkage}

When aneurysm shrinkage was defined as any decrease in any dimension and no increase in any of the other dimensions, the detection rate for aneurysm shrinkage was $3.9 \%$ (Fig. 2). Specifically, the detection rates of aneurysm shrinkage by observers A, B, and C were $2.1 \%, 3.4 \%$, and $5.2 \%$, respectively $(\mathrm{p}=0.52)$. When considering the 4 different MRI modalities, there was no significant difference in interobserver agreement for aneurysm shrinkage $(\mathrm{p}=$ 0.19 for $1.5-\mathrm{T}$ TOF, $\mathrm{p}>0.99$ for $1.5-\mathrm{T} \mathrm{Gd}, \mathrm{p}>0.99$ for 3.0$\mathrm{T}$ TOF, and $\mathrm{p}=0.32$ for $3.0-\mathrm{T} \mathrm{Gd}$ ). The detection rates of aneurysm shrinkage for each MRI modality were $7.2 \%$ for $1.5-\mathrm{T}$ TOF, $1.4 \%$ for $1.5-\mathrm{T} \mathrm{Gd}, 0.29 \%$ for $3.0-\mathrm{T} \mathrm{TOF}$, and $0.28 \%$ for $3.0-\mathrm{T} \mathrm{Gd}(\mathrm{p}=0.35)$.

\section{Detection Rate of Pseudo-Enlarged Aneurysms}

When aneurysms were defined as pseudo-enlarged (i.e., the observer noted an increase in at least 1 aneurysmal dimension and a decrease in at least another dimension), the detection rate of the pseudo-enlargement of aneurysms was $40.6 \%$. Specifically, the detection rates for observers A, B, and C were $49.0 \%, 48.3 \%$, and $26.0 \%$, meaning that there was no significant difference between observer $A$ versus $B(p>0.99)$ but significant differences between observer $A$ versus $C(p=0.002)$ and observer $B$ versus $C(p$ $=0.003)$. When considering the 4 different MRI modalities, there was a significant difference $(p=0.002)$ among observers when using 1.5-T TOF $(\mathrm{p}=0.002)$ and 3.0-T Gd $(\mathrm{p}=0.03)$, but not when using 1.5-T Gd $(\mathrm{p}=0.15)$ and 3.0-T TOF $(p=0.87)$. For $1.5-\mathrm{T}$ TOF, there was no significant difference between observer A versus $B(p=0.06)$ or observer $\mathrm{B}$ versus $\mathrm{C}(\mathrm{p}=0.34)$, but there was a significant difference between observer A versus $C(p=0.001)$. For 3.0-T Gd, there was no significant difference between observer A versus $B(p=0.24)$ or observer $A$ versus $C$ $(p=0.32)$, but there was a significant difference between observer $B$ versus $C(p=0.02)$.

When averaging the 3 observers, the detection rates of pseudo-enlarged aneurysms for each MRI modality were $44.9 \%$ for $1.5-\mathrm{T}$ TOF, $37.1 \%$ for $1.5-\mathrm{T} \mathrm{Gd}, 48.6 \%$ for $3.0-\mathrm{T}$ TOF, and $34.7 \%$ for $3.0-\mathrm{T} \mathrm{Gd}(\mathrm{p}=0.30)$.

\section{Relationship Between the Detection Rate of Aneurysm Enlargement and MRI Modality}

The mean detection rates of aneurysm enlargement for the 3 observers using each MRI modality were $46.4 \%$ for 1.5-T TOF, $61.4 \%$ for $1.5-\mathrm{T} \mathrm{Gd}, 48.6 \%$ for $3.0-\mathrm{T}$ TOF, and $62.5 \%$ for $3.0-\mathrm{T} \mathrm{Gd}$. According to the Fisher exact test, there was no significant difference in the detection rates of aneurysm enlargement between MRI modalities ( $\mathrm{p}=$ 0.11 ), even though these detection rates increased slightly from 1.5-T TOF to 3.0-T Gd.

The detection rates for small enlargement (e.g., 0.4-1 $\mathrm{mm}$ ) had a tendency to increase when using $1.5-\mathrm{T} \mathrm{Gd}$ when compared with 1.5-T TOF $(\mathrm{p}=0.04)$. This was not the case when using $3.0 \mathrm{~T}$ to detect small enlargement 
TABLE 4. Results of the Fisher exact test with the paired-comparison test for interobserver variability in the detection rates of aneurysm enlargement

\begin{tabular}{|c|c|c|c|c|c|c|c|}
\hline \multirow[b]{2}{*}{ Modality } & \multicolumn{3}{|c|}{ Detection Rate } & \multirow[b]{2}{*}{$p$ Value } & \multicolumn{3}{|c|}{ p Value* } \\
\hline & Observer A & Observer B & Observer C & & Observer A vs B & Observer A vs C & Observer $B$ vs $C$ \\
\hline Total & 49.0 & 46.1 & 66.7 & 0.009 & 0.77 & 0.02 & 0.005 \\
\hline 1.5-T TOF & 29.2 & 47.6 & 62.5 & 0.06 & NA & NA & NA \\
\hline 1.5-T Gd & 50.0 & 59.1 & 79.2 & 0.09 & NA & NA & NA \\
\hline 3.0-T TOF & 50 & 45.5 & 50.0 & 0.91 & NA & NA & NA \\
\hline 3.0-T Gd & 66.7 & 45.8 & 75.0 & 0.13 & NA & NA & NA \\
\hline
\end{tabular}

$\mathrm{NA}=$ not applicable.

All values are shown as percentages unless indicated otherwise.

* Determined using the paired-comparison test.

(e.g., 0.4-1 mm). The detection rate of 3.0-T TOF was not statistically different when compared with that of 3.0-T $\mathrm{Gd}(\mathrm{p}=0.19)$. These details are described in Supplemental Fig. 1A-L.

\section{Relationship Between the Detection Rate of Aneurysm Enlargement and Any Increase in Any Aneurysm Dimension}

Supplemental Fig. 2A-D shows the relationship between the detection rates of aneurysm enlargement and increases in the sizes of any aneurysm dimension using the different MRI modalities. As incremental enlargement increased, the detection rates for aneurysm growth increased $(\mathrm{R}=0.71$ for $1.5-\mathrm{T}$ TOF, $\mathrm{R}=0.46$ for $1.5-\mathrm{T} \mathrm{Gd}$, $\mathrm{R}=0.69$ for $3.0-\mathrm{T} \mathrm{TOF}$, and $\mathrm{R}=0.56$ for $3.0-\mathrm{T} \mathrm{Gd}$ ) and the detection rates of aneurysm shrinkage decreased $(\mathrm{R}=$ -0.55 for $1.5-\mathrm{T}$ TOF, $\mathrm{R}=-0.40$ for $1.5-\mathrm{T} \mathrm{Gd}, \mathrm{R}=-0.45$ for 3.0-T TOF, and $\mathrm{R}=-0.51$ for 3.0-T Gd). As mentioned above, the detection rates of small enlargement (e.g., 0.4$1 \mathrm{~mm}$ ) had a tendency to increase when using $1.5-\mathrm{T} \mathrm{Gd}$ compared with 1.5-T TOF (Supplemental Fig. 2A and B).

\section{Relationship Between the Detection Rate of Aneurysm Enlargement and Aneurysm Location}

Supplemental Fig. 3A-H shows the relationship between the detection rates of aneurysm enlargement and aneurysm location. In left ICA-ophthalmic artery aneurysms, the detection rates of aneurysm enlargement were relatively low for all MRI modalities (Supplemental Fig. $3 \mathrm{D})$. On the other hand, there were high detection rates for aneurysm enlargement of the aneurysms of the left terminus of the ICA and cavernous portion of the right ICA using all MRI modalities (Supplemental Fig. 3F and H). For anterior communicating artery and BA aneurysms, the detection rates of both aneurysm enlargement and shrinkage did not show major changes regardless of the MRI modality (Supplemental Fig. 3A and G).

\section{Discussion}

Aneurysm enlargement is considered predictive of aneurysm instability and progression to rupture. ${ }^{6,9,10,15}$ To reliably assess the individual and agreement rates of accurately detecting intracranial aneurysm enlargement, we performed this study using flow phantom models and gen- erally used MRI modalities. The aims of this study were to determine 1) whether the observers could accurately detect any increase in aneurysm dimension using different reading protocols; 2) the minimum incremental increase that can be reliably detected with a high agreement rate; 3 ) the effect of different imaging machines and MRI sequences (TOF vs Gd) on the detection rate; and 4) whether the location of the aneurysm affects the detection rate.

The results of our study suggest that 1) aneurysm dimensions that were increased in the phantom models were detected very reliably by the individual observers, and the agreement rate among the 3 observers was significantly high; 2) aneurysm dimensions that were kept fixed in the phantom models were occasionally miscalculated by the individual observers; 3 ) the detection rate of at least 1 increase in any aneurysm dimension did not depend on the choice of MRI modality; 4) the location of the aneurysm affected the detection rate of aneurysm enlargement; and 5) different measurement protocols did not affect the detection rate of at least 1 increase in any aneurysm dimension for each MRI modality ( $\mathrm{p}=0.31$ ), even though these rates increased slightly from 1.5-T TOF to 3.0-T Gd.

To our knowledge, this is the first study in the literature to identify the smallest incremental aneurysm enlargement that could be reliably detected using different MRA and reading protocols by expert neuroradiologists. The observers consistently and blindly were able to reliably detect incremental growth, even aneurysm dimension increases as small as $0.4 \mathrm{~mm}$. This was a surprise, and counterintuitive, because we expected that due to the voxel size of the generally used MRI resolution the smallest incremental enlargement that would be reliably detected would be at least $0.7-0.8 \mathrm{~mm}$. Any enlargement less than $0.7-0.8 \mathrm{~mm}$ would fall in the range of noise of resolution and therefore could not be expected to be reliably and consistently detected by the 3 observers who used 2 different protocols to determine aneurysm size.

However, the 3 observers occasionally misread the aneurysm dimensions that were maintained at the same size in the 3 different phantoms. Therefore, the margin error of misreading size was within $0.1-0.3 \mathrm{~mm}$ in most of the misread aneurysm dimensions. This leads to speculation that the aneurysm dimensions of our phantom models and our reading protocols can be read within a range of error of $\pm 0.3 \mathrm{~mm}$. Therefore, any changes $\geq 0.4 \mathrm{~mm}$ to an aneu- 
rysm dimension were detected by our observers, especially using 1.5-T MRA with Gd, 3-T TOF MRA, and 3-T MRA with Gd. The use of 1.5-T TOF MRA exacerbated the misreading. Thus, based on our data, 3.0-T imaging may provide the best assessment of size variation. If using 1.5-T MRA, the additional use of Gd improved the detection of size variation in our model. Additionally, in both MR machines (1.5 T and 3.0 T), detecting aneurysm enlargement was better when using Gd than TOF. In this study, because water was circulated in the phantoms instead of blood, we suspected that the signal on TOF MRA might have been lower compared with the signal that would be obtained in patients; hence, the performance of TOF MRA in terms of detecting small aneurysm enlargement might have been underestimated in our study. On the other hand, the use of Gd might enhance the resolution of the aneurysmal lumen and lead to better measurement of different aneurysm dimensions, especially for small aneurysms.

In our study, we found that the location of the aneurysm affected the detection of aneurysm enlargement ( $p$ $<0.0001)$. One potential explanation for this difference is that the aneurysm neck and dome in the terminus segment of the ICA aneurysm could be isolated from its parent artery much easier than the other aneurysms. This observation highlights the fact that the geometry of the aneurysm, its neck, and its parent artery could affect the detection of aneurysm enlargement. Another potential source of the influence of aneurysm location is that there is more or less inflow and more or less disturbed flow within the aneurysm depending on the inflow pattern.

\section{Clinical Importance of Detecting Growth}

Due to the high correlation between aneurysm growth and increased risk of aneurysm rupture, the American Heart Association/American Stroke Association, in their guidelines published in 2015 on the management of unruptured intracranial aneurysms, strongly recommended aneurysm treatment if growth is documented..$^{19}$ This recommendation highlights the importance of reliably documenting aneurysm growth using different imaging modalities.

\section{Limitations of the Current Study}

There are limitations to our study. The first limitation is the use of phantom aneurysms instead of human aneurysms. The disadvantages of this technique are 1) the lack of brain tissue around the aneurysm and bony structures, and 2$)$ the presence of many aneurysms $(n=8)$ in each phantom model. The advantages of this technique are 1) the ability to control the incremental growth in the direction needed, and 2) the ability to limit the number of MR angiograms needed for calculations because of the presence of 8 aneurysms in each phantom model. The second limitation is that the flow dynamics in these phantom models are different from those in human aneurysms; therefore, this could influence the results. The flow used in these models was continuously steady and not pulsatile. In addition, the sensitivity of detecting aneurysm growth might be elevated because artifacts, such as patient motion and swallowing, were ignored in the phantom study. Furthermore, water was introduced as a fluid instead of blood.
However, human studies are very difficult to perform due to difficulties in controlling for incremental aneurysm enlargement.

\section{Conclusions}

The detection rates and interobserver agreement rates were very high for aneurysm enlargement of $0.4 \mathrm{~mm}$ to $2 \mathrm{~mm}$. The detection rate of at least 1 increase in any aneurysmal dimension did not depend on the choice of MRI modality or measurement protocols. Use of Gd improved the accuracy of the measurements. Aneurysm location may influence the accuracy of detecting enlargement. Further studies are warranted to validate these findings and improve the accuracy of detecting aneurysmal enlargement.

\section{Acknowledgments}

This work was supported by funding from the Nakatani Foundation. We thank Faith L. Vaughn for her assistance with the final editing of the manuscript.

\section{References}

1. Anxionnat R, Bracard S, Ducrocq X, Trousset Y, Launay L, Kerrien E, et al: Intracranial aneurysms: clinical value of $3 D$ digital subtraction angiography in the therapeutic decision and endovascular treatment. Radiology 218:799-808, 2001

2. Bor ASE, Rinkel GJE, van Norden J, Wermer MJH: Longterm, serial screening for intracranial aneurysms in individuals with a family history of aneurysmal subarachnoid haemorrhage: a cohort study. Lancet Neurol 13:385-392, 2014

3. Brinjikji W, Zhu YQ, Lanzino G, Cloft HJ, Murad MH, Wang $Z$, et al: Risk factors for growth of intracranial aneurysms: a systematic review and meta-analysis. AJNR Am J Neuroradiol 37:615-620, 2016

4. Fahrig R, Fox AJ, Lownie S, Holdsworth DW: Use of a Carm system to generate true three-dimensional computed rotational angiograms: preliminary in vitro and in vivo results. AJNR Am J Neuroradiol 18:1507-1514, 1997

5. Forbes G, Fox AJ, Huston J III, Wiebers DO, Torner J: Interobserver variability in angiographic measurement and morphologic characterization of intracranial aneurysms: a report from the International Study of Unruptured Intracranial Aneurysms. AJNR Am J Neuroradiol 17:1407-1415, 1996

6. Greving JP, Wermer MJ, Brown RD Jr, Morita A, Juvela S, Yonekura M, et al: Development of the PHASES score for prediction of risk of rupture of intracranial aneurysms: a pooled analysis of six prospective cohort studies. Lancet Neurol 13:59-66, 2014

7. Hirai T, Korogi Y, Arimura H, Katsuragawa S, Kitajima M, Yamura M, et al: Intracranial aneurysms at MR angiography: effect of computer-aided diagnosis on radiologists' detection performance. Radiology 237:605-610, 2005

8. Inoue T, Shimizu H, Fujimura M, Saito A, Tominaga T: Annual rupture risk of growing unruptured cerebral aneurysms detected by magnetic resonance angiography. J Neurosurg 117:20-25, 2012

9. Ishibashi T, Murayama Y, Urashima M, Saguchi T, Ebara M, Arakawa H, et al: Unruptured intracranial aneurysms: incidence of rupture and risk factors. Stroke 40:313-316, 2009

10. Jayaraman MV, Mayo-Smith WW, Tung GA, Haas RA, Rogg JM, Mehta NR, et al: Detection of intracranial aneurysms: multi-detector row CT angiography compared with DSA. Radiology 230:510-518, 2004

11. Juvela S, Poussa K, Porras M: Factors affecting formation 
and growth of intracranial aneurysms: a long-term follow-up study. Stroke 32:485-491, 2001

12. Kouskouras C, Charitanti A, Giavroglou C, Foroglou N, Selviaridis P, Kontopoulos V, et al: Intracranial aneurysms: evaluation using CTA and MRA. Correlation with DSA and intraoperative findings. Neuroradiology 46:842-850, 2004

13. Matsubara S, Hadeishi H, Suzuki A, Yasui N, Nishimura $\mathrm{H}$ : Incidence and risk factors for the growth of unruptured cerebral aneurysms: observation using serial computerized tomography angiography. J Neurosurg 101:908-914, 2004

14. Mehan WA Jr, Romero JM, Hirsch JA, Sabbag DJ, Gonzalez RG, Heit JJ, et al: Unruptured intracranial aneurysms conservatively followed with serial CT angiography: could morphology and growth predict rupture? J Neurointerv Surg 6:761-766, 2014

15. Morita A, Kirino T, Hashi K, Aoki N, Fukuhara S, Hashimoto $\mathrm{N}$, et al: The natural course of unruptured cerebral aneurysms in a Japanese cohort. N Engl J Med 366:2474-2482, 2012

16. Raghavan ML, Ma B, Harbaugh RE: Quantified aneurysm shape and rupture risk. J Neurosurg 102:355-362, 2005

17. Schneiders JJ, Marquering HA, Antiga L, van den Berg R, VanBavel E, Majoie CB: Intracranial aneurysm neck size overestimation with 3D rotational angiography: the impact on intra-aneurysmal hemodynamics simulated with computational fluid dynamics. AJNR Am J Neuroradiol 34:121128,2013

18. Sonobe M, Yamazaki T, Yonekura M, Kikuchi H: Small unruptured intracranial aneurysm verification study: SUAVe study, Japan. Stroke 41:1969-1977, 2010

19. Thompson BG, Brown RD Jr, Amin-Hanjani S, Broderick JP, Cockroft KM, Connolly ES Jr, et al: Guidelines for the management of patients with unruptured intracranial aneurysms: a guideline for healthcare professionals from the American Heart Association/American Stroke Association. Stroke 46:2368-2400, 2015

20. van Rooij SB, van Rooij WJ, Sluzewski M, Sprengers ME: Fenestrations of intracranial arteries detected with 3D rotational angiography. AJNR Am J Neuroradiol 30:13471350,2009

21. van Rooij WJ, Sprengers ME, de Gast AN, Peluso JP, Sluzewski M: 3D rotational angiography: the new gold standard in the detection of additional intracranial aneurysms. AJNR Am J Neuroradiol 29:976-979, 2008

22. Villablanca J, Duckwiler G, Jahan R, Tateshima S, Martin NA, Frazee J, et al: Natural history of asymptomatic unruptured cerebral aneurysms evaluated at CT angiography: growth and rupture incidence and correlation with epidemiologic risk factors. Radiology 269:258-265, 2013

23. Westerlaan HE, van Dijk JMC, Jansen-van der Weide MC, de Groot JC, Groen RJM, Mooij JJA, et al: Intracranial aneurysms in patients with subarachnoid hemorrhage: CT angiography as a primary examination tool for diagnosis-systematic review and meta-analysis. Radiology 258:134-145, 2011 (Erratum in Radiology 260:612, 2011)

24. White PM, Wardlaw JM, Easton V: Can noninvasive imag- ing accurately depict intracranial aneurysms? A systematic review. Radiology 217:361-370, 2000

25. Wiebers DO, Whisnant JP, Huston J III, Meissner I, Brown RD Jr, Piepgras DG, et al: Unruptured intracranial aneurysms: natural history, clinical outcome, and risks of surgical and endovascular treatment. Lancet 362:103-110, 2003

26. Yoon DY, Lim KJ, Choi CS, Cho BM, Oh SM, Chang SK: Detection and characterization of intracranial aneurysms with 16-channel multidetector row CT angiography: a prospective comparison of volume-rendered images and digital subtraction angiography. AJNR Am J Neuroradiol 28:6067, 2007

\section{Disclosures}

The authors report no conflict of interest concerning the materials or methods used in this study or the findings specified in this paper.

\section{Author Contributions}

Conception and design: Hasan, Nakagawa, Raghavan, Woo, Huston, Cloft, Wintermark, Torner, Brown. Acquisition of data: Hasan, Nakagawa, Nagahama, Policeni, Raghavan, Dillard, Schumacher, Sarathy, Dlouhy, Wilson, Allan, Huston, Cloft, Wintermark, Torner, Brown. Analysis and interpretation of data: Hasan, Nakagawa, Nagahama, Policeni, Raghavan, Dillard, Schumacher, Sarathy, Dlouhy, Wilson, Allan, Huston, Cloft, Wintermark, Torner, Brown. Drafting the article: Hasan, Nakagawa, Nagahama, Policeni, Raghavan, Dillard, Schumacher, Sarathy, Dlouhy, Wilson, Allan, Huston, Cloft, Wintermark, Torner, Brown. Critically revising the article: Hasan, Nakagawa, Nagahama, Policeni, Raghavan, Dillard, Schumacher, Sarathy, Dlouhy, Wilson, Allan, Huston, Cloft, Wintermark, Torner, Brown.

Reviewed submitted version of manuscript: Hasan, Nakagawa, Nagahama, Policeni, Raghavan, Dillard, Schumacher, Sarathy, Dlouhy, Wilson, Allan, Huston, Cloft, Wintermark, Torner,

Brown. Approved the final version of the manuscript on behalf of all authors: Hasan. Statistical analysis: Hasan, Nakagawa, Nagahama, Policeni, Raghavan, Dillard, Schumacher, Sarathy, Dlouhy, Wilson, Allan, Huston, Cloft, Wintermark, Torner, Brown.

Administrative/technical/material support: Hasan, Nakagawa, Woo, Huston, Cloft, Wintermark, Torner, Brown. Study supervision: Hasan, Nakagawa, Woo, Huston, Cloft, Wintermark, Torner, Brown.

\section{Supplemental Information}

Online-Only Content

Supplemental material is available with the online version of the article.

Supplemental Figures. https://thejns.org/doi/suppl/10.3171/ 2017.9.JNS171811.

\section{Correspondence}

David M. Hasan: University of Iowa Hospitals and Clinics, Iowa City, IA.david-hasan@uiowa.edu. 